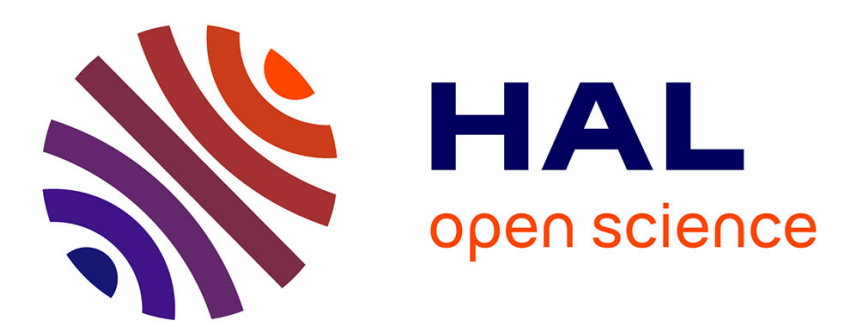

\title{
Single molecule microscopy reveals key physical features of repair foci in living cells
}

\author{
Judith Miné-Hattab, Mathias Heltberg, Marie Villemeur, Chloé Guedj, \\ Thierry Mora, Aleksandra Walczak, Maxime Dahan, Angela Taddei
}

\section{To cite this version:}

Judith Miné-Hattab, Mathias Heltberg, Marie Villemeur, Chloé Guedj, Thierry Mora, et al.. Single molecule microscopy reveals key physical features of repair foci in living cells. 2020. hal-02988483

\section{HAL Id: hal-02988483 \\ https://hal.science/hal-02988483}

Preprint submitted on 5 Nov 2020

HAL is a multi-disciplinary open access archive for the deposit and dissemination of scientific research documents, whether they are published or not. The documents may come from teaching and research institutions in France or abroad, or from public or private research centers.
L'archive ouverte pluridisciplinaire HAL, est destinée au dépôt et à la diffusion de documents scientifiques de niveau recherche, publiés ou non, émanant des établissements d'enseignement et de recherche français ou étrangers, des laboratoires publics ou privés. 
Single molecule microscopy reveals key physical features of repair foci in living cells.

Judith Miné-Hattab ${ }^{1,2, *}$, Mathias Heltberg ${ }^{1,2,3}$, Marie Villemeur ${ }^{1,2}$, Chloé Guedj ${ }^{1,2}$, Thierry Mora $^{3}$, Aleksandra M. Walczak ${ }^{3}$, Maxime Dahan ${ }^{1,4}$, Angela Taddei ${ }^{1,2, *}$

${ }^{1}$ Institut Curie, PSL Research University, CNRS, UMR3664, F-75005 Paris, France.

${ }^{2}$ Sorbonne Université, Institut Curie, CNRS, UMR3664, F-75005 Paris, France.

3 Laboratoire de physique de l'Ecole Normale Supérieure, PSL University, CNRS, Sorbonne Université, Université de Paris.

${ }^{4}$ Institut Curie, PSL Research University, CNRS, UMR168, F-75005 Paris, France.

* co-corresponding authors

\begin{abstract}
In response to double strand breaks (DSB), repair proteins accumulate at damaged sites, forming membrane-less sub-compartments or foci. Here we explored the physical nature of these foci, using single molecule microscopy in living cells. Rad52, the functional homolog of BRCA2 in yeast, accumulates at DSB sites and diffuses $\sim 6$ times faster within repair foci than the focus itself, exhibiting confined motion. The Rad52 confinement radius coincides with the focus size: foci resulting from 2 DSBs are twice larger in volume that the ones induced by a unique DSB and the Rad52 confinement radius scales accordingly. In contrast, molecules of the single strand binding protein Rfa1 follow anomalous diffusion similar to the focus itself or damaged chromatin. We conclude that while most Rfa1 molecules are bound to the ssDNA, Rad52 molecules are free to explore the entire focus possibly reflecting the existence of a liquid droplet around damaged DNA.
\end{abstract}




\section{INTRODUCTION}

The cell nucleus contains membrane-less sub-compartments inside which specific proteins are more concentrated than elsewhere in the nucleus ${ }^{1-3}$. Such regions of high local protein concentration, called "foci", are hypothesized to help proteins coordinate and collectively perform their function ${ }^{2,4-7}$. The formation of these foci at the right place in the nucleus and within a well-defined time window is essential for the functioning of the cell. Here, we focus on repair sub-compartments formed in response to double strand breaks (DBS) ${ }^{8}$. DNA repair is an essential process for preserving genome integrity. Among the different kinds of DNA damages, DSBs are the most genotoxic ${ }^{9}$. Failure to repair such lesions leads to genomic instability or cell death, and mutations in DNA repair genes lead to diseases such as Werner, Bloom and other cancer predisposition syndromes ${ }^{10,11}$. Eukaryotic organisms use mainly two major mechanisms to repair DSBs: non-homologous end-joining (NHEJ) and homologous recombination (HR) ${ }^{12}$. HR occurs primarily in S/G2 phase cells and uses an undamaged homologous DNA sequence as a template for copying the missing information ${ }^{13}$. The biochemistry and the genetics of DSB repair by HR have been extensively investigated in vitro ${ }^{14,15}$ and in vivo ${ }^{16,17}$. HR is orchestrated by mega-Dalton multi-protein complexes of 500 to 2000 proteins that colocalize with the DSB 18. These protein centers can be visualized in living cells as fluorescent foci using tagged HR proteins. Among the proteins occupying these centers are the enzymes of the highly conserved Rad52 epistasis group, including Rad51, Rad52, Rad54 ${ }^{19}$. When a DSB forms, the 5 ' ends of the DNA break are resected by nucleases to yield 3' single-stranded DNA (ssDNA) tails that are rapidly coated by the RPA complex. The Rad52 protein, the functional analog of human BRCA2 in yeast, then stimulates the removal of RPA and recruits the recombinase Rad51 to the ssDNA tail on which it polymerizes ${ }^{19}$. The Rad51-ssDNA complex, called a nucleo-filament, has the capacity to search and identify a region of homology and to promote strand invasion of the homologous duplex DNA ${ }^{20}$. Once homology is found, the invading strand primes DNA synthesis of the homologous template, ultimately restoring genetic information disrupted at the DSB. How repair foci and more generally membrane-less sub-compartments are formed, maintained and disassembled at time scales relevant for their biological function remains unknown. Several models are intensively debated in the literature to understand the nature of membrane-less sub-compartments ${ }^{2,21-26}$.

Here, we present new insights into the dynamics and the nature of repair foci using Single Particle Tracking (SPT) and Photo Activable Localization Microscopy (PALM) in Saccharomyces cerevisiae cells. Watching how proteins move and interact within a living cell is crucial for better understanding their biological mechanisms. SPT is a powerful technique that makes these observations possible by taking 'live' recordings of individual molecules in a cell at high temporal and spatial resolution $(50 \mathrm{~Hz}, 30 \mathrm{~nm})^{27,28}$. Based on the way individual molecules move in vivo, SPT allows for i) sorting proteins into subpopulations characterized by their apparent diffusion coefficients, ii) quantifying their motion, iii) estimating residence times in specific regions of the nucleus, iv) and testing the existence of a potential attracting or repelling molecules within distances smaller than the diffraction limit. To complement this approach, PALM allows for measuring the position of molecules at $30 \mathrm{~nm}$ resolution to establish density maps of the molecules of interest.

Using SPT and PALM in the presence or absence of DSB, we have accessed the dynamics, for the first time at the single molecule level, of 2 repair proteins: $\operatorname{Rad} 52$ and the ssDNA-binding protein Rfa1, a sub-unit of the RPA complex. We find that inside repair foci, Rad52 molecules are surprisingly mobile: they diffuse an order of magnitude faster than Rfa1, the whole focus itself, and damaged DNA, indicating that most of Rad52 molecules are not 
bound to damaged DNA. Instead, Rad52 explores the volume of the focus through confined motion and exhibits a sharp change of diffusion coefficient when entering or escaping foci. In response to multiple DSBs, Rad52 form larger foci than the ones formed upon a single DSB leading to a larger confinement radius for individual Rad52 molecules that scales with focus size. Our statistical analysis of single molecule trajectories reveals the existence of an attractive potential maintaining Rad52 molecules within a focus. Altogether, our results using single molecule microscopy indicate that while Rfa1 diffuses similarly to damaged DNA, Rad52 motion exhibits physical properties consistent with diffusion within a Liquid-Liquid Phase Separated droplet.

\section{RESULTS}

\section{Rad52 exhibits 2 diffusive behaviors in the absence of DSB}

We first investigated the mobility of individual Rad52 molecules in vivo in the absence of DNA damage by SPT. To image Rad52 without altering its endogenous expression level, we generated haploid cells expressing the endogenous Rad52 fused to Halo (Figure 1A, S1 and Methods). Prior to visualization on a PALM microscope (see Methods), exponentially growing cells were incubated with fluorescent and fluorogenic JF646, a dye emitting light only once bound to Halo ${ }^{29}$. We used a low concentration of JF646 allowing for the observation of individual molecules ${ }^{30}$ (Figure S2). Rad52-Halo bound to JF646 (Rad52-Halo/JF646) were visualized at $20 \mathrm{~ms}$ time intervals $(50 \mathrm{~Hz})$ in 2-dimensions during 1000 frames until no signal was visible. A typical individual cell is shown in Figure 1B. After detection and tracking (see Methods), we calculated density and displacement maps of Rad52 molecules (Figure 1). Since Rad52 tracking is performed in 2-dimensions, molecules are observable as long as they stay within the focal plan representing a z-section of about $400 \mathrm{~nm}^{31}$ (Figure 1C). After examining 23 cells in S/G2 phase of the cell cycle, we obtained an exponentially decreasing distribution of trace lengths with an average trajectory length of 7.5 frames, the longest trace reaching 65 frames $(1.3 \mathrm{~s})$ (Figure 1D). Since the half-life time of JF646 is $2.1 \mathrm{~s}$ (Figure S3), the short trace lengths observed here are due to molecules moving out of the observable z-section and not the photo bleaching of the JF646 dyes.

To estimate the apparent diffusion coefficient of Rad52 in the absence of DSB, we calculated their displacement histogram ${ }^{32-34}$. This method allows us to test whether Rad52 molecules exhibit a single diffusive regime across the nucleus or if they exist in several subpopulations characterized by distinct diffusion coefficients (see Methods). By fitting the displacement histogram with 1- and 2-population fits, we observed that Rad52 exhibits 2 distinct diffusive behaviors (Figure 1E-F, $\mathrm{p}=0.0001$ and $\mathrm{p}=0.67$, two-sided Kolmogorov-Smirnoff (KS) test for the 1 and 2 population fits respectively, see Methods). We obtained $D_{1 / \mathrm{S}-\mathrm{G} 2}=1.16 \pm 0.08 \mu \mathrm{m}^{2} / \mathrm{s}$, $\mathrm{D}_{2 / \mathrm{S}-\mathrm{G} 2}=0.28 \pm 0.02 \mu \mathrm{m}^{2} / \mathrm{s}$ as the best-fit diffusion coefficients (see Methods), with the fraction of slow molecules being $0.62 \pm 0.04$. To accurately estimate the apparent diffusion coefficient of Rad52, we then calculated the displacement histograms for several frame rates $(20,40,60$, 80 and $100 \mathrm{~ms}$ time intervals) and fitted these experimental histograms with a 2-population fit (Figure 1G). When mobility is observed at larger time intervals, mean square displacement per unit time decreases, which stems from the confinement of molecules within the nuclear space (we checked that confined diffusion explained the trend better than anomalous diffusion, see Figure S4). Fitting these points with a straight line, we extracted the diffusion coefficients to 
be $\mathrm{D}_{1 / \mathrm{S}-\mathrm{G} 2}=1.16 \pm 0.06 \mu \mathrm{m}^{2} / \mathrm{s}, \mathrm{D}_{2 / \mathrm{S}-\mathrm{G} 2}=0.29 \pm 0.02 \mu \mathrm{m}^{2} / \mathrm{s}$, which agree with the measurements obtained from the displacement histogram with $\Delta \mathrm{t}=20 \mathrm{~ms}$ (Figure $1 \mathrm{G}-\mathrm{H}$ ).

To investigate the origin of the 2 Rad52 populations observed in the nucleus in the absence of DBS, we first asked whether the existence of a slower population of Rad52 is due to its transient association with RPA present at replication forks during $S$ phase. To test this hypothesis, we measured Rad52 mobility in G1 cells. We obtained similar displacement histograms and diffusion coefficients in $\mathrm{G} 1$ as in $\mathrm{S} / \mathrm{G} 2$ cells (Figure 1I, $1 \mathrm{~J}$ and $1 \mathrm{~K}, \mathrm{D}_{1, \mathrm{G} 1}=1.08 \pm$ $0.07 \mu \mathrm{m}^{2} / \mathrm{s}, \mathrm{D}_{2, \mathrm{G} 1}=0.27 \pm 0.03 \mu \mathrm{m}^{2} / \mathrm{s}$ ) with similar proportions. Thus, $\operatorname{Rad} 52$ diffusion does not change significantly during the cell cycle and the 2 observed Rad52 populations cannot be explained by free versus Rad52 molecules transiently associated with the replication fork. We then examined if the 2 observed populations could correspond to the monomeric and multimeric forms of Rad52 observed in vitro ${ }^{35,36}$. We calculated the predicted diffusion coefficient of Rad52 monomers and multimers using the Stokes-Einstein equation (see Methods). For that, we first estimated the dynamic viscosity inside yeast nuclei by measuring the mobility of free NLS-Halo/JF646 (Figure S5). We found a diffusion coefficient of DfreeHalo-NLS $=1.90 \pm 0.06$ $\mu^{2} / \mathrm{s}$, consistent with previous studies by FRAP (Larson et al., 2011). From this measurement, we extracted a dynamic viscosity in the yeast nucleus of $0.122 \pm 0.004$ Pa.s. Using this measured viscosity, the predicted diffusion coefficients of Rad52 monomers and multimers are $D_{\text {mon- }}$ omer $=1.20 \pm 0.04 \mu \mathrm{m}^{2} / \mathrm{s}$ and $D_{\text {multimer }}=0.28 \pm 0.01 \mu \mathrm{m}^{2} / \mathrm{s}$ (see Methods), consistent with the 2 populations $\mathrm{D}_{1}=1.16 \pm 0.08$ and $\mathrm{D}_{2}=0.28 \pm 0.02$ obtained experimentally. Thus, our measurements of Rad52 diffusion by SPT are consistent with the existence of a mixed population of Rad52 inside nuclei, $2 / 3$ being multimers.

\section{Inside foci, Rad52 exhibits a slower diffusion coefficient and a confined motion}

We next investigated how Rad52 diffusion is affected in response to DSB. A single DSB was induced at an I-SceI target site inserted at the LYS2 locus in a strain harboring Rad52Halo (Figure 2A and Methods). Of note, in this setting one can follow only the first step of HR, as no donor sequence is available in the genome. Cells were observed $2 \mathrm{~h}$ after inducing the expression of the endonuclease I-SceI driven by the inducible GAL promoter. In these conditions, the I-SceI site was cleaved in $95 \%$ of the cells ${ }^{37}$ leading to the formation of Rad52 foci ${ }^{38}$. During the second hour of DSB induction, fluorogenic JF646 were added similarly to the previous experiment without DSB and cells were imaged with the same illumination conditions as in the absence of DSB. Inside foci, we observe at most 1 detection of Rad52-Halo/JF646 per frame during the movie, a necessary condition to avoid mislinking when tracking single molecules (Figure S2B). Unlike the experiment in the absence of DSB, the Rad52 density map exhibits a strong accumulation of molecules corresponding to the repair focus in G2/S cells. S/G2 cells harboring a Rad52 focus were selected for further analysis (Figure 2B). As shown by the displacement map (Figure 2B, right panel), molecules localized in Rad52 foci cover shorter distances in a $20 \mathrm{~ms}$ step and can be tracked for longer. Thus, following DSB, the histogram of trace lengths presents a longer tail than before damage, due to Rad52 molecules diffusing slowly inside the focus (Figure 2C).

To estimate the apparent diffusion coefficient of Rad52 in the presence of a single DSB, we tested a 1, 2 and 3-population fit of the Rad52 displacement histograms (Figure 2D). For the 3 populations, we obtained the best fitted values of $\mathrm{D}_{1}=1.17 \pm 0.06, \mathrm{D}_{2}=0.24 \pm 0.04$ and 
finally $\mathrm{D}_{3}=0.054 \pm 0.007 \mu \mathrm{m}^{2} / \mathrm{s}$ (Figure $2 \mathrm{E}, \mathrm{p}=0.0001, \mathrm{p}=0.27$ and $\mathrm{p}=0.67$, two-sided Kolmogorov-Smirnoff (KS) test for the 1, 2 and 3 population fits respectively). Even though the two-population fit provided an acceptable p-value, the 3-population fit allows for recovering the same 2 populations previously observed in the absence of DSB, but supplemented by a third slow population (with diffusivity $\mathrm{D}_{3}$ ). However, the value of $\mathrm{D}_{3}$ obtained in this way is overestimated because of detection noise (in the case of diffusion coefficients $\mathrm{D}<0.1 \mu \mathrm{m}^{2} / \mathrm{s}$, we use mean square displacement analysis allowing us to substrate the noise, see Methods and below). A quick inspection of the spatial distribution of step sizes in the nuclear space indicates that the slowest population is found in the zone where $\operatorname{Rad} 52$ molecules are dense, i.e. within the repair focus (Figure 2B, displacement map). Using a density threshold to separate the trajectories located within the focus from the rest of the nucleus (Figure $2 \mathrm{~F}$, left), we then calculated the displacement histograms of the corresponding trajectories (Figure $2 \mathrm{~F}$, right). We confirm that Rad52 molecules outside foci exhibit similar displacements as in the absence of damage, whereas Rad52 molecules inside the focus are less mobile. We found that the diffusion of Rad52 molecules inside foci is well described by a 1-population fit (Figure S6, p = 0.99, 2sided KS test, see Methods). Like $\mathrm{D}_{3}$, the diffusion coefficient inferred from this fit is affected by detection noise and boundary effects around the focus. To accurately estimate the diffusion coefficient of Rad52 inside the focus accounting for detection noise, and also to measure the nature of Rad52 motion, we used mean squared displacement (MSD) analysis (see Methods). The MSD quantifies the amount of space a molecule explores, and its shape versus time reveals the nature of its movement. This analysis revealed that Rad52 molecules inside foci exhibit a confined motion with a confinement radius $R_{c}=124 \pm 3 \mathrm{~nm}$ and a diffusion coefficient $\mathrm{D}_{\mathrm{Rad5} \text {, in- }}$ side $=0.032 \pm 0.006 \mu^{2} / \mathrm{s}$ (see Methods and Figure $2 \mathrm{G}$ ).

\section{Inside foci, individual Rad52 molecules diffuse faster than the repair focus and the ssDNA-binding protein Rfa1}

We next compared the mobility of individual Rad52 molecules inside foci with the mobility of the focus itself (Figure 3A). After inducing a single DSB for $2 \mathrm{~h}$ in cells harboring Rad52-mMaple, we used high photo-activation illumination to simultaneously activate all Rad52-mMaple and image the repair focus as a single entity (Figure 3B). Using both displacement histograms and MSD analysis (Figure 3C-E and Figure S7), we found that Rad52 molecules diffuse 6.4 times faster than the whole focus $\left(D_{\text {Rad52 inside }}=0.032 \pm 0.006 \mu \mathrm{m}^{2} / \mathrm{s} \mathrm{versus}\right.$ $D_{\text {whole focus }}=0.005 \pm 0.002 \mu \mathrm{m}^{2} / \mathrm{s}$, assuming normal diffusion with a time difference of $20 \mathrm{~ms}$ for the whole focus). Furthermore, the shape of the MSD fits better with an anomalous diffusion with an anomalous exponent of $0.51 \pm 0.05$, as previously reported for damaged chromatin (Mine-Hattab et al, 2017). Thus, our results show that Rad52 molecules are highly mobile inside repair foci.

We then compared the mobility of individual Rad52 molecules with another component of repair foci, the Rfa1 subunit of the single-strand binding factor RPA (Symington et al., 2014) (Figure 4A). Using a strain expressing Rfa1-Halo and experiencing a single I-SceI cut-site, we imaged individual Rfa1-Halo/JF646 $2 \mathrm{~h}$ after DSB induction with the same illumination conditions as Rad52-Halo (Figure 4B). Comparing the displacement histograms of individual Rfa1 and Rad52 molecules, we found that Rfa1 is less mobile than individual Rad52 molecules inside foci (Figure 4C) and exhibits a mobility similar to whole Rad52 foci (Figure 4D). Using both Rfa1 displacement histograms and MSD analysis inside foci, Rfa1 exhibits an apparent normal diffusion coefficient $\mathrm{D}_{\mathrm{Rfa} 1}=0.006 \pm 0.001 \mu \mathrm{m}^{2} / \mathrm{s}$ (Figure $4 \mathrm{E}$, assuming normal diffusion with a time difference of $20 \mathrm{~ms}$ ). Moreover, the MSD of Rfa1 inside foci is similar to the entire 
focus, with an anomalous exponent of $0.56 \pm 0.05$ (Figure 4E and Figure S7). Overall, we found that individual Rad52 diffuses approximately 6 times faster than the whole focus or individual Rfa1 molecules, and exhibits confined diffusion while Rfa1 and the focus follow anomalous diffusion.

\section{Rad52 molecules change diffusion coefficient when entering or escaping repair foci}

The way molecules diffuse around the boundary of membrane-less sub-compartments is crucial for defining their physical properties. We analyzed Rad52 diffusion following DSB in more detail (Figure 5A). First, we estimated the residence time of Rad52 molecules inside foci by calculating the survival probability of Rad52 molecules in a focus. Importantly, by comparing this curve with the bleaching time of the JF646, we checked that JF646 bleaching is not a limiting factor (Figure 5B). We found that the mean residence time of Rad52 inside foci, defined as the integral of the survival probability curve, is $\sim 200 \mathrm{~ms}$ while Rfa1 molecules stay longer ( 560 ms) (Figure 5B).

So far, we analyzed Rad52 trajectories inside and outside repair foci; trajectories crossing focus boundaries were cut. However, traces can be divided in 3 categories: i) traces staying inside repair foci during the time of the acquisition, ii) traces crossing focus boundaries and iii) traces staying outside foci (Figure 5A). Among the first 2 categories of traces, $70 \%$ cross a focus boundary at least once during the 20 s movie, while $30 \%$ stay inside foci, indicating that Rad52 foci are in constant exchange with the rest of the nucleus. As a comparison, only $33 \%$ of Rfa1 molecules cross a focus boundary. Of course, these exchange rates are specific to our acquisition settings.

We then focused only on Rad52 trajectories crossing repair focus boundaries, which we call "travelers" (dotted lines in Figure 5A). To test whether Rad52 molecules change their diffusive behavior when entering or escaping repair foci, we calculated the displacement histograms of these traces, distinguishing portions inside and outside foci (Figure 5A, dotted red and dotted blue traces respectively). We observed that Rad52 molecules change diffusive behavior sharply when crossing the focus boundary. Molecules crossing into foci (category 2) diffuse similarly to molecules staying inside foci, as long as they are in the focus (Figure 5C); when crossing the boundary outward, they start diffusing like molecules outside of foci (Figure 5D). To have a visual representation of this change in diffusion, we displayed the norm of the displacement vectors over time of all travelers, centering all the traces when they cross the boundary (Figure 5E). In other words, Rad52 molecules clearly change diffusion coefficient when entering or escaping repair foci, adopting the diffusive behavior of the surrounding Rad52 molecules in their environment.

\section{Following multiple DSBs, Rad52 confinement scales with focus size}

Following the induction of multiple DSBs, it has been shown in yeast, Drosophila, and human cells that repair sites move and collapse into larger units, or 'clusters' ${ }^{39-45}$, presumably to facilitate DSB repair progression by increasing the local concentration of repair proteins. However, the evidence of DSBs clustering in the literature is often limited by the diffraction limit, and the structure of collapsed DSBs remains unknown. We thus used PALM to investigate the structure of Rad52 foci formed in response to multiple DSBs at the single molecule level. We compared haploid strains harboring a single I-SceI site (Figure 2A) with haploid strains harboring $2 \mathrm{I}$-SceI sites on different chromosomes (Figure 6A and Methods). In both 
cases, DSB(s) were induced for $2 \mathrm{~h}$ and we observed a single focus per nucleus in most cells, as previously described ${ }^{43}$. First, we measured the mobility of the entire Rad52 focus induced by a single DSB versus 2 DSBs, at 20 ms time intervals. Using strains expressing Rad52-mMaple with high photo-activation, we found that both foci exhibit similar mobility (Figure 6B). This is consistent with previous studies reporting no change in chromatin diffusion coefficients when inducing more DSBs despite a dramatic increase in the chromatin confinement radius ${ }^{38}$.

We then compared the size, number of Rad52 molecules and density of foci formed in response to 1 versus 2 DSBs (Figure 6C). Since the focus size is under the diffraction limit, we used PALM in haploid cells expressing Rad52-mMaple. We observed that Rad52 foci are not visible after fixation with classical fixation methods; we performed PALM in living cells ${ }^{46}$ (live PALM) (see Methods). We found that foci induced by 2 DSBs are larger than those induced by a single DSB, $\left(170 \pm 20 \mathrm{~nm}\right.$ versus $116 \pm 20 \mathrm{~nm}$ respectively, $\mathrm{p}=110^{-4}$, WilcoxonMann-Whitney test). In addition, the number of Rad52 molecules forming foci varies from 880 \pm 600 for 1 DSB induced foci to $2300 \pm 811$ for 2 DSBs foci ( $p=810^{-4}$, Wilcoxon-MannWhitney test). Thus, focus density is indistinguishable between foci induced by 1 versus 2 DSBs (Figure 6C, right panel, $\mathrm{p}=0.73$, Wilcoxon-Mann-Whitney test).

We propose 2 views of DSBs clustering illustrated in Figure 6F. In the first view, multiple DSBs cluster and stay close without merging (top figure). In the second, multiple DSBs fuse together and molecules are shared between the different DSBs (bottom figure). While in the first case, Rad52 molecules have similar confinement radius as a single DSB, the second case implies that different foci merge together giving rise to a larger focus where Rad52 molecules explore the entire space. Thus, while the radius of confinement should be unchanged in the first view, it should scale with the focus size in the second one. Here, we found that upon 1 DSB, Rad52 diffuse inside foci with smaller confinement radius (124 $\pm 3 \mathrm{~nm}$ ) compared to foci induced by 2 DSBs (156 $\pm 3 \mathrm{~nm}$ ), supporting the "fusion view" (Figure 6E). Importantly, this confinement radius found by SPT is very close to the mean size of Rad52 foci previously obtained by live PALM. The correlation between focus size and confinement radius indicates that Rad52 is able to explore the entire focus space. To have a visual representation of Rad52 exploration inside foci, we overlaid a reconstruction of a focus with one of the longest Rad52 trajectories contained inside that focus. The reconstruction of the whole focus is obtained by taking all the detections inside it, except the ones belonging to the overlaid trajectory. These detections are represented by a blurred spot using a Gaussian smoothing kernel around each detection. Two typical foci are shown in this representation: a focus formed in response to a single DSB (Figure 6F, left panel) versus a focus induced by 2 DSBs (Figure 6F, right panel). This representation clearly shows that Rad52 explores the focus and that its confinement radius scales with the focus size.

\section{Rad52 shows attractive motion around the focus}

Next, we wanted to estimate whether there was a region of attraction for the molecules of Rad52 inside the focus. For each cell, we considered a set of traces, of which a subset was defined to be inside the focus (Figure 7A). We quantified the radial movement of molecules relative to the center of the focus in terms of the change in the distance to that center between two subsequent time steps, which we denote $\Delta \mathrm{r}$ (Figure 7B). To obtain this measure we needed to estimate the position of the center of the focus. Since we observed that the focus itself was diffusing $\sim 6$ times more slowly than the Rad52 molecules, we approximated the focus as the average particle position in each analyzed trace (see Methods). Concentrating on molecules 
close to the boundary, we identified a region of attraction with an average movement of molecules towards the center of the focus (Figure 7C).

To get a more detailed map of displacements, we plotted the radial movements of all particles as a function of their original position relative to the center for both 1 and 2 DSB's (Figure 7E and 7F). The mean radial movement, outside the focus but close to its boundary, has negative values, which is consistent with an attractive force towards the center of the focus. Inspired by the observation that molecules are attracted to the center of the focus, we computed the difference in the free energy. Using the estimated fraction of molecules belonging to the slow diffusion population (Figure 2), we extracted a change in the Gibbs free energy difference $\mathrm{U}_{0}$ between the inside of the focus and the surroundings. This was done by estimating the increased density of molecules inside the focus using Boltzmann's law for the fraction of slowly diffusing molecules, $p=\frac{e^{-U_{0} / k_{B} T} V_{F}}{V_{0}-V_{F}+e^{-U_{0} / k_{B} T} V_{F}}$, where $V_{F}$ is the volume of the focus, as calculated by the MSD, $V_{0}$ is the volume of the observable frame. Inverting this relation for $\mathrm{U}_{0}$ and injecting the measured values of $p$, $V_{0}$, and $V_{F}$ yields $\frac{U_{0}}{k_{B} T} \approx-5.5$ (Figure $7 \mathrm{G}$ ). However, detection noise (as estimated in Figure 2) and low statistics far away from the focus make it difficult distinguish between a continuous potential and a surface potential.

\section{DISCUSSION}

The nucleus contains membrane-less sub-compartments that form and disassemble according to the needs of the cell at times scales relevant for their biological function. Repair foci provide a powerful example for studying such sub-compartments because it is possible to induce their formation at will in vivo and compare the behavior of repair proteins before and after DSB induction. Following DSB, HR proteins relocalize from a diffuse nuclear distribution to a sub-nuclear focus at the DNA damaged site ${ }^{8}$. To understand this process, here we observed for the first time how Rad52 molecules diffuse inside living nuclei in the presence and in the absence of DSB using single molecule microscopy.

\section{Dynamic behavior of individual Rad52 in living cells}

Tracking single molecules of the repair factor Rad52 at $50 \mathrm{~Hz}$, we observed that in the absence of DSB, Rad52 exhibits 2 distinct diffusive behaviors characterized by apparent diffusion coefficients $D_{1}=1.15 \mu \mathrm{m}^{2} / \mathrm{s}$, and $D_{2}=0.27 \mu \mathrm{m}^{2} / \mathrm{s}$ with a ratio $2 / 1=2 / 3$ (see Table 1 ). These 2 populations were observed both in G1 and S phase ruling out that the slower population corresponds to transient binding of Rad52 at replication forks. Assuming Brownian diffusion, a diffusion coefficient of $1.15 \mu \mathrm{m}^{2} / \mathrm{s}$ implies that Rad52 can go across a yeast nucleus in as little as $700 \mathrm{~ms}$. Tracking free Halo-NLS, we estimate the dynamic viscosity inside yeast nuclei to 0.1 Pa.s, compared to $10^{-3} \mathrm{~Pa}$.s for water at $20^{\circ} \mathrm{C}$ and $6 \mathrm{~Pa} . \mathrm{s}$ for honey. Using the Stokes-Einstein equation, we showed that the 2 diffusion coefficients measured for Rad52 agree with the ones predicted for the monomeric and multimeric forms of Rad52 observed in vitro ${ }^{35,36}$. Thus, single molecule tracking of Rad52 suggests that both forms co-exist in living nuclei, two thirds of them being multimers.

In response to a DSB, Rad52 accumulates at the damaged site forming highly concentrated foci ${ }^{8}$. The mobility of Rad52 within these foci shows an apparent diffusion coefficient of $\mathrm{D}_{\text {Rad52,inside }}=0.032 \pm 0.006 \mu \mathrm{m}^{2} / \mathrm{s}$. The characteristic equilibration time after which the effect 
of boundaries appears is defined by the time at which the MSD curve starts bending and is given by $t_{c}=R_{c}^{2} /(6 \cdot D)$, where $R_{c}$ is the confinement radius and $D$ the diffusion coefficient inside foci ${ }^{33}$. Taken the measured diffusion coefficient and confinement radius, Rad52 molecules start feeling the effect of the focus boundary at only $t_{c}=80 \pm 3 \mathrm{~ms}$ (representing 4 timesteps of $20 \mathrm{~ms}$ ). Whether Rad52 inside foci are monomers or multimers. Assuming a multimeric form, the Rad52 diffusion coefficient measured inside foci would be consistent with a viscosity inside the focus of $1 \pm 0.1 \mathrm{~Pa} . \mathrm{s}^{-1}$, i.e 10 times higher than the viscosity of nucleus before damage.

\section{Different dynamics for Rad52 and Rfa1 within repair foci}

Comparing the dynamics of Rad52 with the single strand binding protein Rfa1, we observed a very different behavior in response to a single DSB. Inside foci, Rfal moves $\sim 6$ times slower than Rad52. Furthermore, individual molecules of Rfa1 and Rad52 exhibit a different type of motion (Figure 5 and S7). Rfa1 molecules inside foci follow anomalous diffusion with an anomalous exponent $(\alpha$ Rfal $=0.56 \pm 0.05)$ consistent with the Rouse model, a behavior similar to the focus itself ( $\alpha_{\text {focus }} 0.51 \pm 0.05$ ), or to damaged chromatin when imaged at the same frequency $(50 \mathrm{~Hz})^{38}$. In contrast, individual Rad52 molecules are highly mobile with a confined motion inside the focus (Figure 4 and Figure S7). Such differences in diffusion coefficient and type of motion between Rad52 and Rfa1 indicate that most of Rad52 molecules are not bound to the Rfa1-coated ssDNA inside foci. We could have expected a second Rad52 population with a slower diffusion coefficient, corresponding to Rad52 bound to Rfa1-coated ssDNA tail. However, we do not observe such a population in our data. Two non-exclusive explanations can account for its absence: i) the fraction of Rad52 bound to ssDNA is extremely small and its contribution does not significantly change the Rad52 displacement histogram. ii) Rad52 is very dynamic and its residence time on ssDNA is smaller than $20 \mathrm{~ms}$ (the time-interval used in our SPT experiments) which could be compatible with its function as a mediator ${ }^{19}$. Rad52, the functional analog of Brca2 in yeast, plays an essential mediator role by removing the RPA complex from the ssDNA tail and by loading Rad51 proteins that form the nucleo-filament. Future experiments at faster time intervals are needed to differentiate between these 2 possibilities.

Rad52 and Rfa1 behaviors also strongly differ at foci boundaries. Rad52 spend less time on average than Rfa1 inside foci (200 ms versus $560 \mathrm{~ms}$ ) and are in constant exchange with the rest of the nucleus unlike Rfa1. Interestingly, when crossing foci boundaries, Rad52 sharply changes diffusion coefficient while Rfa1 molecules remain slow when escaping. In other words, unlike Rfa1, Rad52 diffusive behavior seems determined by the environment where the molecule is at time $t$, and not by its past location.

\section{Rad52, but not Rfa1, diffusion inside foci is consistent with LLPS model}

Different models have been proposed to account for the formation of membrane-less compartments. The simplest model is the binding model where molecules bind and unbind their target sites (i.e. ssDNA for Rfa1). In this case, the local concentration of proteins reflects the number of binding sites, the amount of proteins available and their affinity for their binding sites, without phase separation. If the binding sites are present on chromatin or DNA, these binding sites can be close to each other increasing their local density. In addition, some binding proteins can form bridges between different chromatin loci by creating loops or by stabilizing interactions between distant loci along the chromatin fiber. These interactions can be driven by interactions between chromatin binding proteins or chromatin components. In this model, 
referred to as "Bridging" Model, or Polymer Polymer Phase Separation (PPPS) model ${ }^{2,22}$, the existence of sub-compartments relies on both the binding and bridging properties of these proteins to chromatin. A third scenario is the Liquid-Liquid Phase Separation (LLPS) Model, also referred to as "Droplet Model" ${ }^{23}$. Unlike the binding and the PPPS models, in LLPS, proteins self-organize into liquid-like droplets that grow around a nucleation site allowing certain molecules to become concentrated while excluding others. In this framework, one should distinguish proteins able to initiate a liquid phase separation on their own (scaffold proteins) from proteins concentrating and diffusing freely within this droplet without being responsible for its formation (client proteins) ${ }^{4}$.

Despite a large literature in the field, many in vivo tests commonly used to probe the nature of sub-compartments are extremely phenomenological and insufficient to rule out other possible mechanisms ${ }^{2,24}$. In addition, most of the optical methods used to discriminate models are at the limit of the diffraction and suffer from severe artefacts ${ }^{2,24}$. Overall, there is a lack of solid experimental criteria to confirm one model versus another in living cells, in particular at the microscopic level.

Our results reveal that Rad52, but not Rfa1, motion shares several properties of LLPS models. First, the difference between liquid and other states of matter (like crystal, amorphous solid, liquid crystal or gel) lies in the mobility of the molecules ${ }^{24,47}$. In the LLPS model, even in the presence of a nucleation core, the large majority of proteins are not bound to chromatin ${ }^{2}$. The motion of Rfa1 molecules, which coat ssDNA, is well described by the Rouse model of polymers that characterizes the motion of chromatin ${ }^{48-53}$. In contrast, Rad52 molecules inside foci move faster, exhibiting statistics of confined diffusion with a confinement radius of the same size as the whole focus. Second, LLPS are characterized by a change of motion upon entering or escaping the sub-compartments ${ }^{4,54}$. We observed a sharp change in the diffusion coefficient of Rad52 molecules crossing foci boundary. Third, in the LLPS model, it is energetically more favorable for molecules to stay inside the sub-compartment than to leave because of the presence of an energetic potential maintaining the sub-compartment. Here, we observed the existence of an attractive potential maintaining Rad52 molecules inside the focus even if molecules are not physically bound to a polymer substrate. However, given the noise and the variable size of foci, we cannot currently describe the shape of the potential. Fourth, an important hallmark of LLPS is their ability to fuse, two droplets for radius $\mathrm{R}$ leading to a bigger droplet of doubled volume. In our case, we do not directly visualize the fusion of repair foci, as observed in mammalian cells ${ }^{40,45}$ but, in response to 2 DSBs, most cells show a unique focus, consistent with previous reports ${ }^{13,43}$. We further show that these foci are $1.99 \pm 0.2$ larger in volume than those formed by a single DSB (156 $\pm 3 \mathrm{~nm}$ versus $124 \pm 3 \mathrm{~nm}$ respectively) but present similar Rad52 densities as measured by live PALM. Moreover, focus sizes measured by PALM agree with the confinement radius of individual Rad52 molecules inside foci, showing the ability of Rad52 to explore the whole sub-compartment. Thus, our results indicate that upon 2 DSBs, Rad52 foci do not merely cluster, but form a focus of size consistent with the fusion of 2 foci inside which molecules explore the entire larger sub-compartment. Finally, in LLPS, increasing protein amounts should increase the focus size while concentrations remain the same inside foci and in the nucleoplasm ${ }^{2,22}$. In the case a LLPS is formed but the molecule observed is not the main species driving the LLPS, the concentration inside foci and in the background increases linearly with over-expression of the observed molecule. Here, we found that upon different levels of Rad52 over-expression, the background concentration increases (Figure S8) suggesting that Rad52 might not be the driving molecule responsible for the LLPS formed at the damaged site. In the future, it will be important to measure the size of Rad52 foci at super resolution for several levels of Rad52 upon over-expression. 


\section{Working model for Rad52 foci}

Summing up all our observations, we propose the following model. In response to a DSB, Rad52 diffusion is not altered in the nucleus, thus Rad52 molecules likely do not exhibit a strong collective or directive motion toward the damaged DNA site. They rather arrive by simple diffusion at the DSB where they bind ssDNA coated by the RPA complex. The focus is then formed by a small seed of Rad52 molecules bound to the ssDNA tail, around which a large cloud of Rad52 rapidly diffuse exploring the whole focus. Similar to LLPS, Rad52 foci are dense but surprisingly dynamic: Rad52 molecules inside foci are $\sim 6$ times more mobile than ssDNA-binding proteins Rfa1 with permanent exchange with the rest of the nucleus. When escaping foci, Rad52 molecules change their diffusion behavior outside foci, suggesting that their diffusive behavior is defined by its environment. Rad52 molecules are maintained inside a focus by an attractive force towards the focus center. The existence of such attractive potential supports a model where DNA is dispensable to maintain the phase separation once a given saturating concentration of multivalent binder molecules is reached. Overall, our results at the single molecule level reveal that Rad52 foci exhibit many features of LLPS at the microscopic level, consistent with recent studies using macroscopic criteria both in yeast and in human cells 21,25 . Also consistent with Rad52 molecules diffusing within a droplet associated with the damage site is our observation that Rad52 foci could not be observed after fixation while foci formed by other proteins fused to fluorescent protein remains visible in the same conditions.

Our results provide a quantitative measurement of Rad52 behavior once the focus is formed and future studies will be necessary to investigate Rad52 foci formation. Over-expressing Rad52 at different levels suggest that Rad52 acts as a client of the LLPS but does not directly drive its formation.

In the future, single molecule microscopy combined with genetics will be a powerful method to shed light on the role of mutants affecting diffusion, residence time, focus size and the type of motion. In particular, sumoylation might have an impact on the formation and the physical nature of Rad52 foci ${ }^{55}$. More generally, applying single molecule microscopy to other biological processes may reveal that nuclear sub-compartments with very similar aspects and sharing some macroscopic features of LLPS are in fact formed by other alternative mechanisms. For example, silencing sub-compartments resemble Rad52 foci: they fuse and disassemble in specific metabolic conditions. However, single molecule microscopy reveals that Sir3 proteins diffuse very differently than Rad52 and do not behave as expected in a LLPS (Sridhar et al., in preparation). In human cells, Herpes Simplex Virus appears to share many properties commonly attributed to LLPS, but SPT measurements have recently shown that they are formed through a distinct compartmentalization mechanism ${ }^{54}$. As the list of proteins that can undergo phase separation is growing in the literature ${ }^{56,57}$, it will be essential to confirm the nature of sub-compartments using in vivo single molecule approaches. It also becomes necessary to define solid criteria and observables at the microscopic level to distinguish between different models (Heltberg et al., in preparation) and to develop alternative models of membrane-less sub-compartments. 


\section{ACKNOWLEDGEMENTS:}

The authors thank Vu Nguyen and Carl Wu for fruitful exchanges on the use of JF dyes and for sharing plasmids. We thank Antoine Coulon, Maxime Woringer, for their comments on the manuscript, as well as Bassam Hajj for his help during the construction of the PALM set-up. We also thank Mickael Garnier for his help on image analysis and Gaizka Le Goff for his help on preliminary experiments performed during his L3 internship.

A.T. team was financially supported by funding from the Labex DEEP (ANR-11-LABEX-0044 DEEP and ANR-10-IDEX-0001-02 PSL), from the ANR DNA-Life (ANR-15-CE12-0007), ANR-12-PDOC-0035-01, Q-life (ANR-17-CONV-0005) Fondation pour la Recherche Médicale (DEP20151234398), and CNRS grant 80prime PhONeS, the authors greatly acknowledge the PICT-IBiSA@Pasteur Imaging Facility of the Institut Curie, member of the France Bioimaging National Infrastructure (ANR-10-INBS-04).

\section{FIGURES LEGEND:}

Figure 1: Rad52 diffusion observed at the single molecule level, in the absence of DNA damage.

(A) Design of the experiment. Haploid yeast strain harboring Rad52 endogenously fused to Halo and incubated with fluorogenic JF646 dyes are visualized by Single Particle Tracking (SPT). Individual Rad52-Halo/JF646 are tracked at $20 \mathrm{~ms}$ time intervals $(50 \mathrm{~Hz})$, movies are 1000 frames long.

(B) Typical S/G2 haploid cells harboring Rad52-Halo coupled with JF646 (Rad52Halo/JF646). From left to right: transmission image; time-projection of a typical SPT acquisition (see Method); Rad52 detections: each spot represents a single detection of Rad52Halo/JF646, the color map indicates the number of Rad52 neighbors inside a $50 \mathrm{~nm}$ radius disk; Rad52 traces: each line represents the trajectory of a detection, the color map indicates the distance covered in $20 \mathrm{~ms}$. The bar scale represents $1 \mu \mathrm{m}$. This typical nucleus exhibits 682 detections and 129 traces.

(C) Schematic of SPT experiment: individual molecules are tracked in 2-dimensions. The blue rectangle represents the observable $\mathrm{z}$-section.

(D) Distribution of tracks length of Rad52-Halo/JF646 in the absence of DNA damage. The histogram combines $23 \mathrm{~S} / \mathrm{G} 2$ phase cells representing 964 traces (mean length of 7.5 fames), and 6252 displacements of $20 \mathrm{~ms}$.

(E) Probability Density Function (PDF) of Rad52-Halo/JF646 molecules of haploid S/G2-phase cells in the absence of DNA damage. The time interval is $20 \mathrm{~ms}$. Red: 2-population fit; dashed purple: slow population; dashed orange: fast population; Black: 1-population fit.

(F) Cumulative Density Function (CDF) of Rad52-Halo/JF646 molecules in haploid S/G2-phase cells in the absence of DNA damage. Dashed green line: data; Red: 2-population fit; Black: 1-population fit. The p-values are indicated in parenthesis (see Methods).

(G) Probability Density Function (PDF) of Rad52-Halo/JF646 molecules in haploid S/G2-phase cells in the absence of DNA damage, for time-points spaced by 20, 40, 60, 80, 100 and $120 \mathrm{~ms}$. The lines show the 2-population fit performed on each PDF.

(H) Diffusion coefficient D $\left(\mathrm{mm}^{2} / \mathrm{s}\right)$ obtained from the fits shown in Figure $1 \mathrm{G}$ for each $\Delta t$. The fits (dotted lines) represent the expected fraction calculated from simulations (see Methods). 
(I) Typical G1 cell harboring Rad52-Halo/JF646. Left: transmission image; right: timeprojection of a typical SPT acquisition (1000 frames).

(J) Displacement histogram of S/G2 cells (blue) versus $\mathrm{G} 1$ cells (red).

(K) Probability Density Function (PDF) of Rad52-Halo/JF646 molecules in haploid G1 cells in the absence of DNA damage. Black: 1-population fit; Red: 2-population fit; dashed purple: slow molecule; dashed orange: fast molecules.

\section{Figure 2: Rad52 diffusion at the single molecule level in the presence of a single I-SceI induced DSB.}

(A) Design of the experiment. We induced a single DSB in haploid cells harboring Rad52 endogenously fused to Halo as well as an I-SceI cut site inducible under galactose promoter. The DSB is induced for $2 \mathrm{~h}$ and fluorogenic JF646 dyes are added to the medium during the last hour of incubation prior visualization by SPT. Individual Rad52-Halo/JF646 are tracked at $20 \mathrm{~ms}$ time intervals $(50 \mathrm{~Hz})$, during 1000 frames. Only cells harboring a Rad52 focus are analyzed.

(B) Typical S/G2-phase haploid cells harboring Rad52-Halo/JF646. From left to right: transmission image; time-projection of a typical SPT acquisition; Rad52 detections: each spot represents a single detection of Rad52-Halo/JF646, the color map indicates the number of Rad52 neighbors inside a $50 \mathrm{~nm}$ radius disk; Rad52 traces: each line represents the trajectory of a detection, the color map indicates the distance in $\mu \mathrm{m}$ covered in $20 \mathrm{~ms}$. The bar scale represents $1 \mu \mathrm{m}$. This particular nucleus exhibits 682 detections and 129 traces.

(C) Distribution of tracks length of Rad52-Halo/JF646 in the presence and the absence of a single DSB. The histogram combines $27 \mathrm{~S} / \mathrm{G} 2$ phase cells, all of them harboring a Rad52 focus, representing 1061 traces (mean length of 8 fames), and 8495 displacements of $20 \mathrm{~ms}$ time-intervals.

(D) Probability Density Function (PDF) of Rad52-Halo/JF646 molecules in haploid S/G2-phase cells in the presence of a single DSB. The time interval is $20 \mathrm{~ms}$. Green: Rad52 data (27 cells, 1061 trajectories); Black: 1-population fit; Red: 2-population fit; Yellow: 3-population fit.

(E) Cumulative Density Function (CDF) of Rad52-Halo/JF646 molecules in haploid S/G2-phase cells in the presence of single DSB. Dashed green line: data; Black: 1-population fit; Red: 2-population fit; Yellow: 3-poulations fit. The p-values are indicated in parenthesis (see Methods).

(F) Left: density map of a typical nucleus following the induction of a single DSB. The nucleus is divided in 2 zones based on a density threshold: the Rad52 focus (highlighted in red) and the rest of the nucleus (highlighted in blue). Right: displacements histograms of trajectories contained inside foci (red) versus outside foci (green). If a trajectory crosses the focus boundary, it is cut into 2 parts: the part inside and the part outside of the focus. The blue histogram represents the displacement of Rad52 molecules in the absence of DSB (shown in Figure 1E).

(H) Mean Square Displacement (MSD) curve of Rad52/Halo/JF646 molecules inside foci. The dotted line shows a fit of the MSD with a confined model (see Methods).

\section{Figure 3: Individual Rad52 molecules are more mobile than the whole focus}

(A) Schematic of the experiment: to shade in light the internal dynamics of Rad52, we compare the mobility of the entire Rad52 foci, shown in light red, with the mobility of individual Rad52 molecules located inside foci, in red. The mobility of the entire Rad52 
focus is measured using a strain harboring Rad52-mMaple and a single I-SceI DSB at LYS2 (see Method). We used high photo-activation illumination to simultaneously activate all Rad52-mMaple and image the entire foci as a single large spot. Rad52 foci were tracked at $20 \mathrm{~ms}$ time-intervals in 2 dimensions.

(B) Typical image of a haploid cell harboring a Rad52-mMaple focus after a $2 \mathrm{~h}$-induction of a single DSB. Left: transmission image; Middle: typical frame of a movie in which we see the whole focus; Right trajectory of the whole focus after analysis. The bar scale represents $1 \mu \mathrm{m}$.

(C) Displacements histogram of the entire Rad52 foci (grey), compared with individual Rad52-Halo located inside foci (red, same data shown in Figure $2 \mathrm{~F}$ in red). Both displacements are measured after $2 \mathrm{~h}$ of galactose induction at $20 \mathrm{~ms}$ time intervals in 2 dimensions. $14 \mathrm{~S} / \mathrm{G} 2$ cells harboring a Rad52 focus are analyzed, representing 131 traces (mean length 24 frames), and 3015 translocations of 20 ms time-intervals.

(D) PDF of the entire Rad52 foci calculated for 20, 40 and $60 \mathrm{~ms}$ time intervals. Plain lines represent a 1-population fit of the PDF.

(E) MSD of the entire Rad52 foci (green) versus individual Rad52 molecules located inside foci (red, same data as Figure 2G). The MSD of individual Rad52 molecules inside foci is fitted with a confined model (dotted line) while the MSD of the entire Rad52 focus is fitted using an anomalous model (see Methods).

\section{Figure 4: Rad52 and Rfa1 diffusion strongly differ inside foci}

(A) Simplified view of a DSB repaired by HR where RPA is shown.

(B) Typical S/G2-phase haploid cells harboring Rfa1-Halo/JF646 after $2 \mathrm{~h}$ if galactose induction. From left to right: transmission image; Time-projection of the whole movie; Rfa1 density map: each spot represents a single detection of Rfa1-Halo/JF646, the color map indicates the number of Rfa1 neighbors inside a $50 \mathrm{~nm}$ radius disk; Rfa1 displacement map: each line represents the trajectory of a detection, the color map indicates the distance in $\mathrm{mm}$ covered in $20 \mathrm{~ms}$. The bar scale represents $1 \mu \mathrm{m}$.

(C) Displacement histograms of individual Rfa1 molecules (yellow) and individual Rad52 molecules (red) inside foci. The time interval is $20 \mathrm{~ms}$. 29 S/G2 cells were analyzed, representing 621 traces (mean length of 12.4 frames) and 7095 displacement of $20 \mathrm{~ms}$ time intervals.

(D)Displacement histograms of individual Rfa1 molecules inside foci (yellow) and whole Rad52 foci (grey). The time interval is $20 \mathrm{~ms}$.

(E) MSD of individual Rfa1moelcuels inside foci (yellow), individual Rad52 molecules inside foci (red, same data as Figure 3E) and the whole Rad52 focus (green, same data as Figure 3E). MSDs of Rfa1 molecules and the whole Rad52 focus are fitted with an anomalous model while the MSD of individual Rad52 is fitted with a confined model.

\section{Figure 5: Rad52 diffusion coefficient changes when molecules enter and escape repair foci}

A) Illustration of the 3 categories of Rad52 traces observed in response to a single DSB in the nucleus: i) traces staying inside repair foci (plain red), ii) traces crossing foci boundaries (dotted lines), with red-dotted lines for the part inside foci and blue-dotted lines for the part outside; iii) traces staying outside of the foci (plain blue). 
B) Survival probability curve of Rad52 molecules inside foci (red), Rfa1 (yellow) and renormalized bleaching curve of the JF646 (black) (see Figure S3).

C) Light red: displacement histogram of traces represented as dotted red lines in Figure 4A (travelers, part inside foci). Dark red: displacement histogram of traces represented in plain red in Figure 4A (traces inside foci).

D) Light blue: displacement histogram of traces represented as dotted blue lines in Figure 4A (travelers, part outside foci). Dark blue: displacement histogram of traces represented in plain blue in Figure 4A (traces outside foci).

E) Left: Step size for traces inside the focus (green), crossing (blue) and outside the focus (red). The $\mathrm{x}$-axis is squeezed so all traces take the same space, in order to visually compare the step sizes. Above: traces starting inside and ending outside. Below: traces starting outside and ending inside the focus. Right: Bar plot showing the estimated diffusion coefficient calculated from all the traces.

\section{Figure 6: Rad52 foci in the presence of multiple DSBs}

A) Design of the experiment. We induced 2 I-SceI DSBs (at LYS2 and HIS3) in haploid cells harboring Rad52-Halo (see Methods). The DSB is induced for $2 \mathrm{~h}$ and fluorogenic JF646 dyes are added to the medium during the last hour of incubation prior visualization by SPT. Individual Rad52-Halo/JF646 are tracked at $20 \mathrm{~ms}$ time intervals $(50 \mathrm{~Hz})$, during 1000 frames. Only cells harboring a Rad52 focus are analyzed.

B) Displacement histogram whole Rad52 foci: Blue: after the induction of a single DSB (same data as 3C); Red: after the induction of 2 DSBs. Rad52 foci were tracked at 20 $\mathrm{ms}$ time intervals after $2 \mathrm{~h}$ of galactose induction. For the experiments following the induction of 2 DSBs, we examined 14 cells, representing 702 traces (mean length of 9.8 frames) and 6220 displacements of $20 \mathrm{~ms}$ time intervals.

C) Top left: Rad52 Focus size measured for 1 DSB-induced foci versus 2 DSBs-induced foci. We performed live PALM on cells harboring Rad52-mMaple strains (see Methods). Bottom left: Estimation of the number of Rad52 molecules inside 1 versus 2 DSBsinduced foci, measured by live PALM. Right: Rad52 Foci density of 1 versus 2 DSBsinduced foci.

D) Illustration of the "Cluster" and the "Fusion" scenarii in the case of multiple DSBs.

E) MSD of 1 versus 2 DSBs-induced foci (red and blue curves respectively). Dotted lines represented a fit of the experimental MSDs, using a model of confined diffusion.

F) Typical example of a Rad52 trajectory represented in blue. The whole focus is shown in the background using a Gaussian blur of each Rad52 detections contained in this focus. Left: 1 DSB-induced focus; right: 2 DSBs-induced focus.

\section{Figure 7: Rad52 shows attractive motion around the focus}

A) Representative cell, with traces outside the focus (black) and inside the focus (blue). Cell nucleus is estimated from the traces.

B) Schematic showing the definition of the radial displacement $\Delta r$, defined by two subsequent data-points.

C) Sign of the average radial displacement as a function of particle position relative to the focus, averaged over all cells. 
D) Radial displacement $\Delta \mathrm{r}$ versus $\mathrm{r}$ (distance to the center of the focus prior to displacement) over all cells with 1 DSB. Cyan line indicates the mean value. Black line corresponds to the expected movement for free diffusion with diffusion coefficient equal the one inside the focus (assuming the center of focus is exactly known). Red line corresponds to a free diffusion, simulated with same trace length and the same diffusion coefficient as the data traces and the same method for estimating the center of the focus.

E) Same as E) but for cells with 2 DSBs.

\section{MATERIAL AND METHODS}

All strains used in this work are isogenic to RAD5+ W303 derivatives (Supplementary Table S1).

\section{Strain constructions}

Strain harboring Rad52-mMaple ${ }^{58}$ or Rad52-Halo are constructed using crisper-Cas9 technic: the fusion is made at the Rad52 N-terminus with a 48 bases linker (CGTACGCTGCAGGTCGACGGAGCAGGTGCTGGTGCTGGTGCTGGAGCA) or a 15 bases linker (AGTGGAGGCGGAGGT), respectively. For strains harboring Rad52-mMaple, we first built plasmid pAT475 containing the cas9 sequence and a gRNA targeting Rad52. The donor sequence containing the mMaple sequence was produced from plasmid pAT446 (pUM003, kindly provided by Jonas Ries laboratory, EMBL), using primers am1941 and am1942 (see Table S1). Cells were transformed simultaneously with the gRNA-RAD52 plasmid (pAT475) and the donor DNA on -LEU plates. For strains harboring Rad52-Halo, we amplified the HaloTag sequence from the plasmid pAT496 (pBS-SK-Halo-KanMX), kindly provided by the Carl Wu laboratory using primers am2182 and am2402. Cells were co-transformed with the gRNARAD52 plasmid (pAT475) and the donor DNA on -LEU plates. After clones validation by PCR (am384, am1944 and am1947), cells are re-streaked several times on YPD until the loss of the gRNA-RAD52 plasmid.

In Rad52-Halo strains, the pleiotropic drug resistance PDR5 gene was replaced by the $\mathrm{N}$-acetyltransferase (NAT) gene using deletion plasmid collection from the toolbox ${ }^{59}$ and S1/S2 primers: pAT197, am2165 and am2166. The replacement of PDR5 gene with the 3-isopropylmalate dehydrogenase (LEU2) gene was performed by PCR-based gene targeting using pAT297; primers am2609 and am2610. The PDR5 gene deletion was checked by PCR using following primers: am431, am2167, am2168 and am2421.

The functionality of Rad52 in these strains was verified using a dilution assay on plates containing MMS at different concentrations (Figure S1).

\section{Induction of a DSB}

Before microscopy, strains harboring an I-SceI cut site under galactose promoter were pregrown in $\mathrm{SC}$ medium and diluted at $\mathrm{OD}_{600 \mathrm{~nm}}=0.01$ in $\mathrm{SC}+3 \%$ raffinose medium overnight. In the morning, cells were diluted at $\mathrm{OD}_{600 \mathrm{~nm}}=0.2$ in $\mathrm{SC}+3 \%$ raffinose medium until they reach $\mathrm{OD}_{600 \mathrm{~nm}}=0.4$. To induce the DSB, $2 \%$ galactose were then added directly into the tube for $2 \mathrm{~h}$, and fluorogenic JF646 (Grimm et al., 2015) were added directly into the tube during the last hour of galactose induction. 


\section{Sample preparation for the microscopy:}

A key aspect of fluorescent experiment is the choice of a suitable fluorophore. Several parameters have to be taken into account, depending on the experiments: specificity of the dyes, density of labeling, brightness, photo-stability. For SPT, in which we want to follow individual molecules as long as possible, we choose fluorogenic HaloTag JF646 dyes ${ }^{29}$ for their brightness and specificity. Indeed, fluorogenic JF646 dyes have the ability to emit light only when they are bound to a Halo molecule and no unspecific signal was observed. Cells are prepared in dark tubes and incubated with $5 \mathrm{nM}$ JF646 during $1 \mathrm{~h}$.

For live PALM, in which we want to observe each Rad52 molecules only once, we used a strain harboring endogenously fused Rad52-mMaple ${ }^{58}$. Unlike the SPT experiments using external dyes, here, all the Rad52 molecules are fused to mMaple, allowing the observation of all Rad52 molecules for quantification of focus size, number of Rad52 molecules and density. Due to the photo-conversion properties of mMaple, it is estimated that $70 \%$ of mMaple can be photo-converted ${ }^{58}$, which is taken into account in our analysis.

\section{Single Particle Tracking:}

We acquired single molecule images on a custom setup based on a Nikon iSPT-PALM inverted microscope. For SPT experiments, we used fluorogenic JF646 dyes excited with a 647-nm laser with a power of $1.9 \mathrm{~kW} / \mathrm{cm}^{2}$ at the sample. The fluorescent signal is captured with an EM-CCD camera (Ixon Ultra 897 Andor) using a 100x/1.45NA (Nikon) objective. Using this objective, the image pixel size was $160 \mathrm{~nm}$. We controlled the microscope with NIS software (Nikon). Experiments were performed at $30^{\circ} \mathrm{C}$ using a Tokai temperature control (STXG-TIZWX-SET) placed at the objective. We detected and connected the spots obtained in the movies with a custom algorithm derived from (Sergé et al., 2008) and we used home-made programs to visualize density and displacements detections. We kept all traces equal or longer than 2 points for further analysis.

\section{Estimation of diffusion coefficients}

Displacement histogram:

Probability Density Functions (PDF) are obtained after normalization of the displacement histograms and fitted with a 1, 2 or 3-population model ${ }^{33,34}$ :

1-population model: $P D F(r, t)=\frac{2 r}{4 D t} \exp \left(\frac{-r^{2}}{4 D t}\right)$, where $D$ is the diffusion coefficient and $r$ is the position at time $t$.

2-population model: $P D F(r, t)=f \frac{2 r}{4 D_{1} t} \exp \left(\frac{-r^{2}}{4 D_{1} t}\right)+(1-f) \frac{2 r}{4 D_{2} t} \exp \left(\frac{-r^{2}}{4 D_{2} t}\right)$, where $D_{1}$ and $D_{2}$ are the diffusion coefficients of sub-population 1 and 2 respectively, and $f$ is the fraction of sub-population 1.

3-population model:

$P D F(r, t)=f_{1} \frac{2 r}{4 D_{1} t} \exp \left(\frac{-r^{2}}{4 D_{1} t}\right)+f_{2} \frac{2 r}{4 D_{2} t} \exp \left(\frac{-r^{2}}{4 D_{2} t}\right)+\left(1-f_{1}-f_{2}\right) \frac{2 r}{4 D_{3} t} \exp \left(\frac{-r^{2}}{4 D_{3} t}\right)$, where $D_{1}, D_{2}$ and $D_{3}$ are the diffusion coefficients of sub-population 1, 2 and 3 respectively, $f_{1}$ and $f_{2}$ are the fractions of sub-population 1 and 2 . 
In order to estimate the diffusion coefficients, we started out with the null hypothesis that traces were described by one diffusion coefficient. We simulated trajectories using the maximum likelihood estimate for the diffusion coefficient and compared the simulated and experimental CDF using a two-sided Kolmogorov-Smirnoff (KS) test to test the one diffusion coefficient hypothesis.

If one diffusion coefficient could not describe the data, we used maximum likelihood to find the two diffusion coefficients and the population fraction diffusing with each coefficient. We used a Metropolis Hastings algorithm to sample the two diffusion coefficients from the posterior distribution. Based on the distribution of the diffusion coefficients we estimated the uncertainty in the extracted parameters.

\section{Criteria to select traces inside repair foci:}

We use 2 methods to select traces inside foci:

i) Since foci are much denser than the rest of the nucleus, we selected traces based on a density threshold. For these traces, we tested a 2-population fit $\left(\mathrm{D}_{\text {inside- } 1}=0.26 \pm\right.$ $0.02 \mu \mathrm{m}^{2} / \mathrm{s}$, and $\mathrm{D}_{\text {inside- } 2}=0.066 \pm 0.004 \mu \mathrm{m}^{2} / \mathrm{s}, \mathrm{p}=0.56,2$-sided KS test), with slow molecules being the large majority $(0.76 \pm 0.04)$. However, the diffusion of fast molecules is similar to $\mathrm{D}_{2}(0.24 \pm 0.04)$, and by removing boundary points, we obtain a very good fit using a 1-population fit $(\mathrm{p}=0.99,2$-sided KS test) inside the focus (data not shown). This analysis is referred as "cropped data" in Table 1.

ii) Since no trace longer than 70 were found in the absence of DSB, we selected traces longer than 70 time-points and assumed that they would be majorly populated by a slow diffusion coefficient. However, since molecules can travel back and forth between the focus and rest of the nucleus, we separated the traces belonging to the slow diffusion coefficient by assaying each displacement a probability based on the slow diffusion coefficient $\mathrm{D}_{3}=0.054$ and removed points with probability less than $1 / 10$ 000. This analysis is refereed as "Traces longer than 70 time-points" in Table 1 .

After selecting traces inside foci with each method, we used MSD analysis to calculate the diffusion coefficient of molecules and decipher the nature of Rad52 motion inside foci (see below). Both methods lead to similar results (see Table 1).

\section{Mean Square Displacement:}

In the case of slow diffusion coefficients ( $D<0.1 \mu \mathrm{m}^{2} / \mathrm{s}$ ), we needed to extract the diffusion coefficient from the experimental noise. For that, we calculated the MSD,

$$
\operatorname{MSD}(n \cdot \Delta t)=\frac{1}{N-n} \sum_{i=1}^{N-n}\left[\left(x_{i+n}-x_{i}\right)^{2}+\left(y_{i+n}-y_{i}\right)^{2}\right]
$$

where $\mathrm{N}$ is the number of points in the trajectory, $(x, y)$ the coordinates of the locus in 2-dimensions and $\Delta t$ the time interval used during the acquisition. To obtain a precise estimation of the confinement radius and the diffusion coefficient, we calculated time-ensemble-averaged MSD over several trajectories, which are simply referred to as "MSD" in the Figures. To check possible artefacts due to the variability between molecules, we also computed individual MSD i.e. MSD calculated from a single trajectory (data not shown). 
When the motion appeared confined, we fit the MSD with $M S D(t)=R_{\infty}^{2}\left(1-e^{-2 d D t / R_{\infty}^{2}}\right)+4 \sigma^{2}$, to extract the experimental noise level $\sigma^{2}$, the radius of confinement $R_{c}=R_{\infty} \sqrt{\frac{2+d}{2}}$, where $d$ is the dimension of the motion, and the diffusion coefficient $\mathrm{D}^{33,47}$.

When the motion is not a simple confinement but is modulated in time and space with scaling properties, it is called anomalous sub-diffusion. In this case, sub-diffusive loci are constrained, but, unlike confined loci, they can diffuse without boundaries and thus reach further targets if given enough time. For sub-diffusive motion, the MSD exhibits a power law and is fitted with $M S D(t)=A t^{\alpha}+\varepsilon$, where $\alpha$, the anomalous exponent, is smaller than $1, A$ is the anomalous diffusion coefficient, and $\varepsilon$ is the noise due to the experimental measurements (Miné-Hattab et al., 2017, p.). For the wild type data, we fitted both functions to the data, and found a better agreement with the confinement fit.

\section{Estimation of the dynamic viscosity and the diffusion coefficient of Rad52 monomers ver- sus multimers:}

To estimate the dynamic viscosity of the nucleus in the absence of DSB, we use Stokes-Einstein equation:

$$
D=\frac{k_{b} T}{6 \pi \eta r}
$$

where $D$ is the diffusion coefficient, $r$ is the radius of a spherical molecule, $\eta$ is the dynamic viscosity, $k_{B}$ the Boltzmann constant and $T$ the absolute temperature.

We used $1.5 \mathrm{~nm}$ and $9 \mathrm{~nm} \pm 1$ for the radius of a $\operatorname{Rad} 52$ monomer and the radius of a $\operatorname{Rad} 52$ multimer ${ }^{35,36}$.

Errors on $D$ and $\eta$ are calculated using error propagations: $\Delta \eta=\left|\frac{\partial D}{\partial \eta}\right| \Delta \mathrm{D}$ and $\Delta D=\left|\frac{\partial \eta}{\partial D}\right| \Delta \eta$.

\section{Simulations :}

In order to extract as much information as possible from the traces, we performed simulations to correctly quantify the effects of: 1) the reduction in the number of observed fast molecules, due to their larger rate to exiting the z-frame undetected than slow molecules, and 2) the reduced diffusion coefficient, due to the collisions with the nucleus boundary.

To understand 1), we simulated 10000 particles for 10 seconds, inside a sphere of $1 \mu \mathrm{m}$ radius, where traces were only measured if the particle were within the window of $|z|<0.15 \mu \mathrm{m}$. We counted the number of detected datapoints, as a function of the size of the time window using time windows of ts $=5-100 \mathrm{~ms}$. We found the number of detected datapoints decayed exponentially for all diffusion coefficients, but the magnitude of the decay rate grew as a function of the real diffusion coefficient. Therefore we took all of the computed decay rates, and fitted them to a power law, giving us an expression for the rate at which the number of observed molecules decays as a function of the time window and the true diffusion coefficient. We do not know the total number of particles in each population and we cannot determine this relation directly. We use this empirical scaling of the relation between the two populations.

In the same manner, we estimated 2) how the diffusion coefficients were affected. We measured the MSD and found that the diffusion coefficient obtained from this measure decayed as a function of the true diffusion coefficient. We directly calculated the diffusion coefficient from the maximum likelihood, and measured the gradient in the increase of the diffusion coefficeint, assuming the considered time window is constant. By doing this we could fit the gradient and determine the expected diffusion coefficient . 


\section{Estimation of radial motion}

We used data cropped to movement inside the focus to estimate radial motion. We used all traces longer than 30 points and estimated the center of the focus by calculating their mean value for each trace in the $\mathrm{x}$-direction and $\mathrm{y}$-direction. We calculated the distance to the center for all points, and defined the change in radius as $\Delta \mathrm{r}$. We grouped datapoints from all traces and compared the statistics with a random walk with no attraction but with a similar diffusion coefficient (dashed lines Figures 7D+E).

\section{Live PALM:}

For live PALM experiments, we used strains harboring Rad52-mMaple as well as 1 or 2 I-SceI cut inductible under galactose. Samples were prepared with living cells and mMaple was excited with a 561-nm laser at power of $6 \mathrm{~kW} / \mathrm{cm}^{2}$ at the sample (max power) as well as a pulsed 405-nm laser to photo-activate mMaple. Movies were performed at $20 \mathrm{~ms}$ time interavals, during 2000 frames (no more spot were detectable at the end of the acquisition). Spots were detected using a custom algorithm derived from ${ }^{60}$ and home-made programs to visualize spots density and quantify foci.

REFERENCES:

1. Meldi, L. \& Brickner, J. H. Compartmentalization of the nucleus. Trends in Cell Biology 21, 701-708 (2011).

2. Miné-Hattab, J. \& Taddei, A. Physical principles and functional consequences of nuclear compartmentalization in budding yeast. Current Opinion in Cell Biology 58, 105-113 (2019).

3. Rippe, K. Dynamic organization of the cell nucleus. Current Opinion in Genetics \& Development 17, 373-380 (2007).

4. Banani, S. F., Lee, H. O., Hyman, A. A. \& Rosen, M. K. Biomolecular condensates: organizers of cellular biochemistry. Nat Rev Mol Cell Biol 18, 285-298 (2017).

5. Cabianca, D. S. et al. Active chromatin marks drive spatial sequestration of heterochromatin in C. elegans nuclei. Nature 569, 734-739 (2019).

6. Chubb, J. R. \& Bickmore, W. A. Considering Nuclear Compartmentalization in the Light of Nuclear Dynamics. Cell 112, 403-406 (2003). 
7. Hübner, M. R. \& Spector, D. L. Chromatin Dynamics. Annu. Rev. Biophys. 39, 471-489 (2010).

8. Lisby, M., Barlow, J. H., Burgess, R. C. \& Rothstein, R. Choreography of the DNA Damage Response: Spatiotemporal Relationships among Checkpoint and Repair Proteins. 15.

9. Iliakis, G. et al. Mechanisms of DNA double strand break repair and chromosome aberration formation. Cytogenet Genome Res 104, 14-20 (2004).

10. Rassool, F. V. DNA double strand breaks (DSB) and non-homologous end joining (NHEJ) pathways in human leukemia. Cancer Letters 193, 1-9 (2003).

11. Thorslund, T. \& West, S. C. BRCA2: a universal recombinase regulator. Oncogene 26, 7720-7730 (2007).

12. Wyman, C. \& Kanaar, R. DNA Double-Strand Break Repair: All's Well that Ends Well. Annu. Rev. Genet. 40, 363-383 (2006).

13. Lisby, M. \& Rothstein, R. Cell Biology of Mitotic Recombination. Cold Spring Harb Perspect Biol 7, a016535 (2015).

14. Kaniecki, K., De Tullio, L. \& Greene, E. C. A change of view: homologous recombination at single-molecule resolution. Nat Rev Genet 19, 191-207 (2018).

15. Miné, J. et al. Real-time measurements of the nucleation, growth and dissociation of single Rad51-DNA nucleoprotein filaments. Nucleic Acids Research 35, 7171-7187 (2007).

16. Lisby, M. \& Rothstein, R. Choreography of recombination proteins during the DNA damage response. DNA Repair 8, 1068-1076 (2009).

17. Sánchez, H. et al. Architectural plasticity of human BRCA2-RAD51 complexes in DNA break repair. Nucleic Acids Research 45, 4507-4518 (2017). 
18. Lisby, M., Rothstein, R. \& Mortensen, U. H. Rad52 forms DNA repair and recombination centers during S phase. Proceedings of the National Academy of Sciences 98, 8276-8282 (2001).

19. Symington, L. S., Rothstein, R. \& Lisby, M. Mechanisms and Regulation of Mitotic Recombination in Saccharomyces cerevisiae. Genetics 198, 795-835 (2014).

20. Bordelet, H. \& Dubrana, K. Keep moving and stay in a good shape to find your homologous recombination partner. Curr Genet 65, 29-39 (2019).

21. Altmeyer, M. et al. Liquid demixing of intrinsically disordered proteins is seeded by poly(ADP-ribose). Nat Commun 6, 8088 (2015).

22. Erdel, F. \& Rippe, K. Formation of Chromatin Subcompartments by Phase Separation. Biophysical Journal 114, 2262-2270 (2018).

23. Hyman, A. A., Weber, C. A. \& Jülicher, F. Liquid-Liquid Phase Separation in Biology. Annu. Rev. Cell Dev. Biol. 30, 39-58 (2014).

24. McSwiggen, D. T., Mir, M., Darzacq, X. \& Tjian, R. Evaluating phase separation in live cells: diagnosis, caveats, and functional consequences. Genes Dev. 33, 1619-1634 (2019).

25. Oshidari, R. et al. DNA repair by Rad52 liquid droplets. Nat Commun 11, 695 (2020).

26. Strom, A. R. et al. Phase separation drives heterochromatin domain formation. Nature 547, 241-245 (2017).

27. Manley, S. et al. High-density mapping of single-molecule trajectories with photoactivated localization microscopy. Nat Methods 5, 155-157 (2008).

28. Oswald, F., L. M. Bank, E., Bollen, Y. J. M. \& Peterman, E. J. G. Imaging and quantification of trans-membrane protein diffusion in living bacteria. Phys. Chem. Chem. Phys. 16, 12625-12634 (2014). 
29. Grimm, J. B. et al. A general method to improve fluorophores for live-cell and single-molecule microscopy. Nat Methods 12, 244-250 (2015).

30. Ranjan, A. et al. Live-cell single particle imaging reveals the role of RNA polymerase II in histone H2A.Z eviction. eLife 9, e55667 (2020).

31. Hansen, A. S. et al. Robust model-based analysis of single-particle tracking experiments with Spot-On. eLife 7, e33125 (2018).

32. Hansen, A. S. et al. Spot-On: robust model-based analysis of single-particle tracking experiments. http://biorxiv.org/lookup/doi/10.1101/171983 (2017) doi:10.1101/171983.

33. Klein, H. L. et al. Guidelines for DNA recombination and repair studies: Cellular assays of DNA repair pathways. Microb Cell 6, 1-64 (2019).

34. Stracy, M. \& Kapanidis, A. N. Single-molecule and super-resolution imaging of transcription in living bacteria. Methods 120, 103-114 (2017).

35. Saotome, M. et al. Structural Basis of Homology-Directed DNA Repair Mediated by RAD52. iScience 3, 50-62 (2018).

36. Shinohara, A., Shinohara, M., Ohta, T., Matsuda, S. \& Ogawa, T. Rad52 forms ring structures and co-operates with RPA in single-strand DNA annealing. Genes to Cells 3, 145156 (1998).

37. Batté, A. et al. Recombination at subtelomeres is regulated by physical distance, doublestrand break resection and chromatin status. EMBO J 36, 2609-2625 (2017).

38. Miné-Hattab, J. \& Rothstein, R. Increased chromosome mobility facilitates homology search during recombination. Nat Cell Biol 14, 510-517 (2012).

39. Aten, J. A. Dynamics of DNA Double-Strand Breaks Revealed by Clustering of Damaged Chromosome Domains. Science 303, 92-95 (2004). 
40. Aymard, F. et al. Genome-wide mapping of long-range contacts unveils clustering of DNA double-strand breaks at damaged active genes. Nat Struct Mol Biol 24, 353-361 (2017).

41. Chiolo, I. et al. Double-Strand Breaks in Heterochromatin Move Outside of a Dynamic HP1a Domain to Complete Recombinational Repair. Cell 144, 732-744 (2011).

42. Krawczyk, P. M. et al. Chromatin mobility is increased at sites of DNA double-strand breaks. Journal of Cell Science 125, 2127-2133 (2012).

43. Lisby, M., Mortensen, U. H. \& Rothstein, R. Colocalization of multiple DNA double-strand breaks at a single Rad52 repair centre. Nat Cell Biol 5, 572-577 (2003).

44. Neumaier, T. et al. Evidence for formation of DNA repair centers and dose-response nonlinearity in human cells. Proceedings of the National Academy of Sciences 109, 443-448 (2012).

45. Schrank, B. R. et al. Nuclear ARP2/3 drives DNA break clustering for homology-directed repair. Nature 559, 61-66 (2018).

46. Izeddin, I. et al. Super-Resolution Dynamic Imaging of Dendritic Spines Using a Low-Affinity Photoconvertible Actin Probe. PLoS ONE 6, e15611 (2011).

47. Lesne, A. Physical analysis of chromatin and chromosome movements : from classical theories to open challenges. 5 .

48. Backlund, M. P., Joyner, R., Weis, K. \& Moerner, W. E. Correlations of three-dimensional motion of chromosomal loci in yeast revealed by the double-helix point spread function microscope. MBoC 25, 3619-3629 (2014).

49. Burnecki, K. et al. Universal Algorithm for Identification of Fractional Brownian Motion. A Case of Telomere Subdiffusion. Biophysical Journal 103, 1839-1847 (2012).

50. Hajjoul, H. et al. High-throughput chromatin motion tracking in living yeast reveals the flexibility of the fiber throughout the genome. Genome Res. 23, 1829-1838 (2013). 
51. Lucas, J. S., Zhang, Y., Dudko, O. K. \& Murre, C. 3D Trajectories Adopted by Coding and Regulatory DNA Elements: First-Passage Times for Genomic Interactions. Cell 158, 339352 (2014).

52. Miné-Hattab, J., Recamier, V., Izeddin, I., Rothstein, R. \& Darzacq, X. Multi-scale tracking reveals scale-dependent chromatin dynamics after DNA damage. MBoC 28, 3323-3332 (2017).

53. Weber, S. C., Spakowitz, A. J. \& Theriot, J. A. Bacterial Chromosomal Loci Move Subdiffusively through a Viscoelastic Cytoplasm. Phys. Rev. Lett. 104, 238102 (2010).

54. McSwiggen, D. T. et al. Evidence for DNA-mediated nuclear compartmentalization distinct from phase separation. elife 8, e47098 (2019).

55. Altmannova, V. et al. Rad52 SUMOylation affects the efficiency of the DNA repair. Nucleic Acids Research 38, 4708-4721 (2010).

56. Mészáros, B. et al. PhaSePro: the database of proteins driving liquid-liquid phase separation. Nucleic Acids Research gkz848 (2019) doi:10.1093/nar/gkz848.

57. You, K. et al. PhaSepDB: a database of liquid-liquid phase separation related proteins. Nucleic Acids Research 48, D354-D359 (2020).

58. McEvoy, A. L. et al. mMaple: A Photoconvertible Fluorescent Protein for Use in Multiple Imaging Modalities. PLoS ONE 7, e51314 (2012).

59. Janke, C. et al. A versatile toolbox for PCR-based tagging of yeast genes: new fluorescent proteins, more markers and promoter substitution cassettes. Yeast 21, 947-962 (2004).

60. Sergé, A., Bertaux, N., Rigneault, H. \& Marguet, D. Dynamic multiple-target tracing to probe spatiotemporal cartography of cell membranes. Nat Methods 5, 687-694 (2008). 

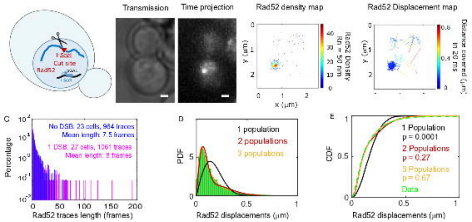

F
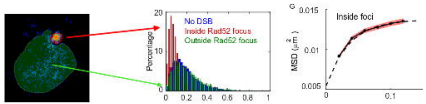

Rec152 dsvacomerts (um) x. $\rightarrow$ 


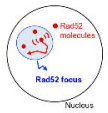

- Wability of inctuidiea

Rat 52 meleures:

- Mooliy or the mals Foct52 lows

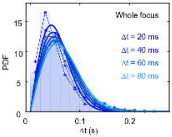

Rad52 tcoue frame 1
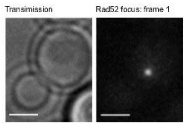

E
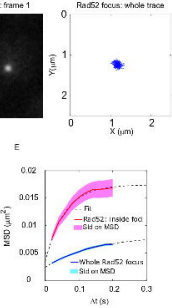

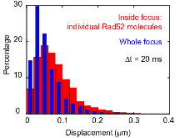

D

$c$ 


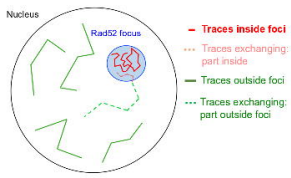

C

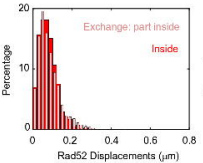

D

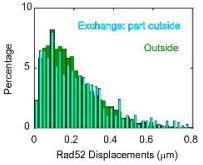

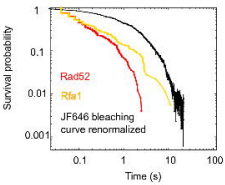

E

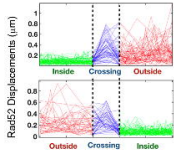

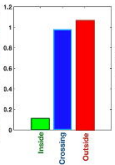




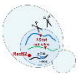

Wobic of Redfe rod

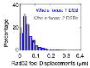

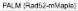

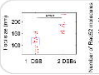

E

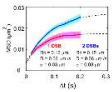

r

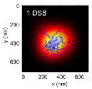

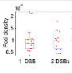

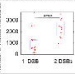

$\therefore$
$\therefore$
$\therefore$ Co 8 ;

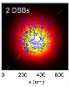

: n-1

\section{Wusta castas as} toe eths.l revery

Wue pe DeB $1 x$

A. als ilhias ivida a syp" tion trat"

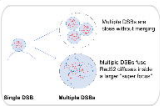



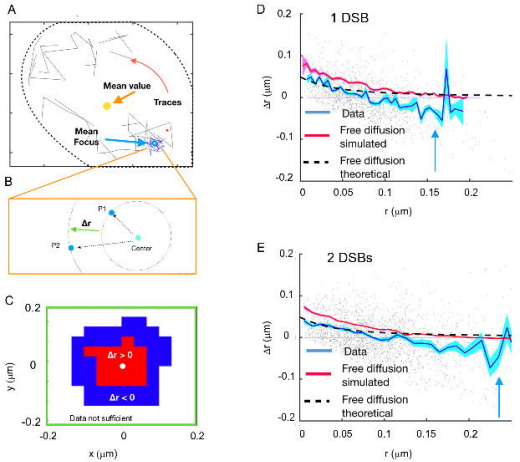